\title{
Effect of High-Energy Ball Milling on the Microstructure of Natural Graphite
}

\author{
J.M. Mendoza-Duarte, R. Martínez-Sánchez, I. Estrada-Guel
}

Centro de Investigación en Materiales Avanzados (CIMAV). Laboratorio Nacional de Nanotecnología, Miguel de Cervantes No. 120, 31109, Chihuahua, Mexico

Graphite (Gr) is recognized as a high strength, low-density material, because of its high strength to mass ratio. Besides has been used extensively as reinforcement materials in epoxy and polymer based composites [1]. Another advantages are its excellent structural stability and constant mechanical performance even at high temperature [2]. Recently, an elevate interest in materials with nanocrystalline grain size (from few to a tens of nanometers) is emerging because a reduction of particle and grain size induces important changes of physical and mechanical properties. Several authors have examined the change in the crystallinity of graphite during ball milling looking for unusual properties for technical applications. Using high-energy ball milling (HEBM) technique, it is possible to produce a fine and homogeneous distribution of particles sizes. Also it causes changes on an atomic level and destruction of the crystal structure by the damage of weak bonds between adjacent atoms.

This work deals with the study of the effect of HEBM over the structure and properties of natural graphite. Pure graphite flakes (-10 mesh and 95.5\% purity) was used as raw material. Gr was milled in a SPEX-8000M mill (under an Ar atmosphere) using a hardened vial with steel balls; keeping a ball to sample ratio of 5:1. Selected milling intensities were $0,1,2,4$ and $16 \mathrm{~h}$. Surface analysis was carried out measuring the BET surface area. The morphology, size and particle distribution of graphite particles were performed with a scanning electron microscope JSM-7201F.

The Fig 1 shows the effect of milling over the morphology and particle size of the graphite particles. It is evident that normal graphite structure was deformed due to impact forces released by the collision of milling media and exhibits a notable signal of defoliation by graphite layers sliding. Experimental results of adsorption-desorption isotherms showed that milled graphite adsorption properties depend of pore characteristics and specific surface area $\left(\mathrm{S}_{\mathrm{sp}}\right)$, both derived from milling.

In Fig. 2 (inner table) it is evident an accentuated $S_{s p}$ increase in the samples as a direct function of milling intensity. Common $S_{\mathrm{sp}}$ values reported in the literature are included between 15 to $70 \mathrm{~m}^{2} / \mathrm{g}$. However, as result of mechanical exfoliation process, the experimental value found of $98.6 \mathrm{~m}^{2} / \mathrm{g}$ demonstrates the efficacy of the process.

[1] S.W. Ip, R. Sridhar, J.M. Toguri, T.F. Stephenson, A.E.M. Warner, Mat. Sci. and Eng. A244 (1998) 31-38.

[2] H. Mayer, M. Papakyriacou, Carbon 44 (2006) 1801-1807.

[3] H. Kwon, M. Estili, K. Takagi, T. Miyazaki, A. Kawasaki. Carbon 47 (2009) 570-577.

[4] The Research was supported by CONACYT (Project No. 169262) and Redes Temáticas de Nanociencias y Nanotecnología (124886). 



Figure 1. SEM micrographs of isolated graphite particles after $0,2,4$ and 8 hours of milling. It is evident the reduction of particle size and an increased level of defoliation with further processing.

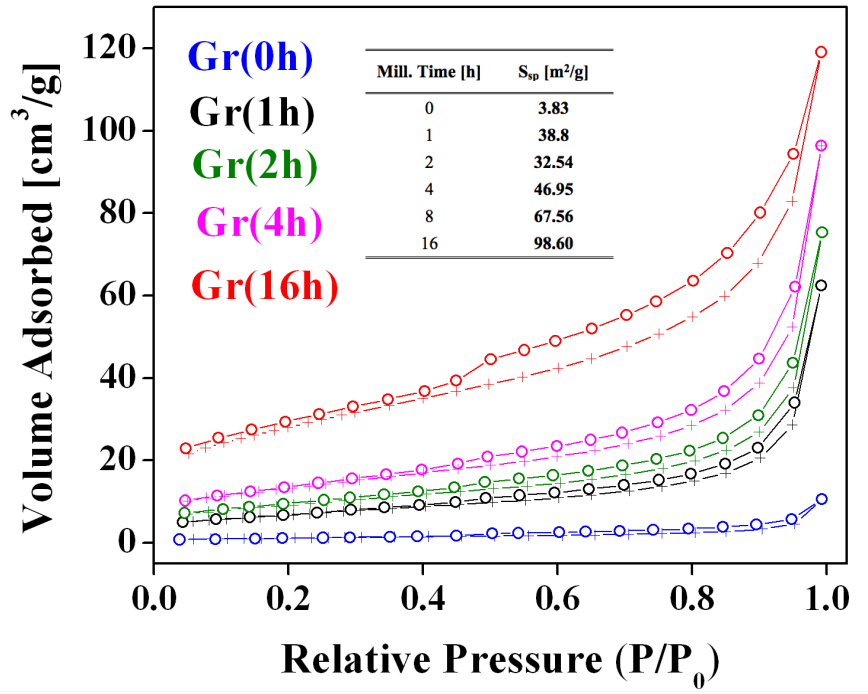

Figure 2. Isotherm curves in $\mathrm{N} 2$ at $77 \mathrm{~K}$. Unmilled graphite curve is classified into a II type, suggesting the existence of macropores. The others consist in IIV type with an important content of micropores. 